\title{
Ejaculatio praecox
}

\section{Wissenslücken bei „zu frühem Kommen“}

- Jeder dritte Teilnehmer (34\%) einer Umfrage von Springer Medizin zum Thema vorzeitiger Samenerguss nannte als häufigste Ursache korrekt eine angeborene Störung im zentralen Serotonin-Transporter-System. Weit mehr als die Hälfte (57\%) der vorwiegend Allgemeinmediziner gab hingegen eine psychische Belastungssituation als Hauptursache an.

Auch die Prävalenz der Erkrankung wurde unterschätzt. Fast drei Viertel (74\%) nannten eine Prävalenz von unter $2 \%$, mehr als jeder dritte (35\%) von unter $10 \%$. Nur jeder fünfte gab die Prävalenz mit 21-30\% korrekt an.

Auch wenn die 210 Teilnehmer bei Ursache und Prävalenz mehrheitlich danebenlagen, hatte dies wenig Auswirkungen auf die Therapieempfehlungen: Rund jeder zweite (54\%) würde seinen Patienten zu Verhaltenstherapien wie "Start-StoppTechnik" oder "Squeeze-Technik" raten und weit mehr als jeder Zweite (59\%) würde ei- ne Pharmakotherapie empfehlen, z. B. mit dem kurz wirksamen SSRI Dapoxetin (als Priligy ${ }^{\circledast}$ speziell in der Indikation Ejaculatio praecox zugelassen).

Hierzu passt, dass gut 40\% der Ärzte in der nicht repräsentativen Umfrage um die Bedeutung des Neurotransmitters Serotonin bei der Kontrolle des Ejakulationsvorgangs wussten. Lokale Therapien wurden von jedem dritten Teilnehmer der von Berlin-Chemie unterstützten Umfrage empfohlen, etwa lidocainhaltige Cremes, jeder dritte würde zum Urologen überweisen.

Die Umfrage ergab außerdem: Mehr als jeder Dritte (37\%) gab an, dass die Sexualanamnese in seiner Praxis regelmäßiger Bestandteil des Patientengesprächs sei. Dieser hohe Anteil zeigt, dass die 210 Teilnehmer dem Thema sexuelle Störungen gegenüber sehr aufgeschlossen sind.

- Michael Hubert

Quelle: Umfrage von Springer Medizin

\section{HIV-Infektion}

\section{„Test and Treat" ist das Gebot der Stunde}

— Seit etwa 2008 gehe der Trend wieder eindeutig in Richtung früher Therapie bei HIV-Infektion, betonte Prof. Frank-D. Goebel, München. So werde in den im Frühjahr aktualisierten Empfehlungen des US-amerikanischen Department of Health and Human Services (DHHS) explizit empfohlen, alle HIV-Infizierten antiretroviral zu therapieren, also auch symptomlose mit hoher CD4-Zellzahl.

Von einer frühen Therapie können auch Kontaktpersonen profitieren, betonte Goebel. Gezeigt wurde dies u.a. in der HPTN052-Studie, an der heterosexuelle Paare teilnahmen, bei denen ein Partner HIV-infiziert war. Dieser wurde entweder sofort antiretroviral behandelt oder erst bei einer CD4-Zellzahl von unter 250 pro Mikroliter.

"Die frühe Behandlung verringerte das Übertragungsrisiko um 96\%", so Goebel. Die Frühtherapie sei damit eine hoch wirk- same Prophylaxe. Auch die Infizierten selbst hatten etwas davon. Bei ihnen traten im Studienzeitraum 41\% weniger HIV-1assoziierte Ereignisse auf, wenn schon bei einer CD4-Zellzahl von 550 pro Mikroliter behandelt wurde.

Insgesamt sei die antiretrovirale Therapie in den letzten Jahren immer besser individualisierbar geworden, betonte Goebel. Gut handhabbare Kombinationspräparate wie Emtricitabin/Tenofovir (Truvada ${ }^{\oplus}$ ) und so genannte Single Tablet Regimen (STR), also komplette antiretrovirale Therapieregime in einer einzigen Tablette zur einmal täglichen Einnahme, wie Efavirenz/Emtricitabin/Tenofovir (Atripla ${ }^{\oplus}$ ) und Rilpivirin/Emtricitabin/Tenofovir (Eviplera $^{\oplus}$ ) haben dazu beigetragen.

\section{- Philipp Grätzel von Grätz}

Quelle: 8. Gilead Campus Infektiologie, Potsdam, August 2012 (Veranstalter: Gilead Sciences)
Kurz notiert

QR-Code führt direkt zum Anwendungsvideo > Der Inhalt des Atemwegspräparates Viani® ${ }^{\circledR}$ bleibt gleich, aber die Diskus-

Umverpackungen

sind nun mit

einem QR-Code

bedruckt. Dieser kann mit Hilfe eines Smartphones gescannt werden. Er leitet weiter zu einem Videoclip im Internet, der demonstriert, wie der Diskus richtig angewendet wird.

\section{GlaxoSmithKline}

Brennen beim Wasserlassen $>$ Jede fünfte erwachsene Frau erleidet pro Jahr eine oder mehrere Infektionen der unteren Harnwege. Wie die empirische antibakterielle Therapie für diese Patientinnen aussehen sollte, haben nationale und internationale Leitlinien und Empfehlungen zusammengefasst. Mittel der Wahl für die unkomplizierte Zystitis der Frau ist z. B. Fosfomycin-Trometamol (Monuril $^{\otimes} 3000$ Granulat). Dieses Antibiotikum hat viele Vorteile: compliancefördernde Einmalgabe, langanhaltende Wirkstoffkonzentration im Urin, Wirksamkeit gegen relevante Uropathogene und auch nach jahrzehntelangem Einsatz eine gute Resistenzsituation. In einer Veröffentlichung der Paul Ehrlich Gesellschaft 2012 weisen in Deutschland E. coli aus Urinisolaten eine Resistenzrate von nur 1,2\% aus. Darüber hinaus ist die Gefahr mikrobiologischer Kollateralschäden für Fosfomycin gering.

Pierre Fabre Pharma

Patientenratgeber zu Atemwegsinfekten $>$ Ein kostenlose Ratgeber informiert Patienten über die Ursachen von Erkältungskrankheiten sowie die Therapie, z. B. mit GeloMyrtol ${ }^{\circledR}$ forte. Sie können die Servicebox per E-Mail bestellen: anforderung@pohlboskamp.de.

Pohl Boskamp 